\title{
Upcoming Events in Pediatric Cardiology Pediatric Cardiology 42-2
}

(c) Springer Science+Business Media, LLC, part of Springer Nature 2021

Please note that due to Covid-19 pandemic, many meetings are being held virtually. Please check organization websites for details.

February 11-14, 2021.

24th Annual Update on Pediatric and Congenital Cardiovascular Disease: Resilience in Pediatric and Congenital Cardiovascular Disease

https://chop.cloud-cme.com/cours e/cours eover view? $\mathrm{P}=0 \& \mathrm{EID}=1103$

March 5-6, 2021

The Children's Cardiomyopathy Foundation (CCF) is hosting the 5th International Conference on Cardiomyopathy in Children

Virtual event

https://www.childrenscardiomyopathy.org/unique/physicianresources/scientific-conference.html

March 20-21, 2021

Congenital Cardiac Anesthesia Society Annual Meeting

Virtual Meeting

https://www.ccasociety.org/meetings/upcoming/

March 30-31, 2021

Artificial Intelligence

AI med Clinical Series: Surgery and Procedural

https://ai-med.io/all-events/clinician-series/surgery-andprocedural/

April 17, 2021

Echocardiographic Assessment of Common Congenital Heart Disease and Coronary Artery Imaging Workshop Virtual Conference

https://www.luriechildrens.org/en/events/annual-chicagosonographer-update/

April 30-May 2, 2021

American Association of Thoracic Surgery, Annual Meeting
Seattle, Washington, USA — Check to see if conference will be changed to a virtual meeting

http://www.aats.org

May 4-5, 2021

The 12th European Meeting on Adult Congenital Heart Disease (EuroGUCH 2021), the annual meeting of the Working Group on Adult Congenital Heart Disease of the European Society of Cardiology

This meeting will be virtually held on the 4th and 5th May 2021

https://www.escardio.org/Congresses-\&-Events/Workinggroup-events/EuroGUCH

May 15-21, 2021

American College of Cardiology, Annual Scientific Session Atlanta, Georgia, USA-Check to see if conference will be changed to a virtual meeting

https://accscientificsession.acc.org/

June 10, 2021

Congenital Cardiac Nurses Association

Virtual Conference 2021_- "Congenital Heart Disease, lockdown and me: Global perspectives of living through a pandemic"

Virtual conference

http://www.ccn-a.co.uk/virtual-conference-10th-june-2021

June 18-21, 2021

American Society of Echocardiography, Annual Scientific Sessions

Boston, Massachusetts, USA-Check to see if conference will be changed to a virtual meeting https://www.asecho.org

June 24-26

CSI Congress Frankfurt

Frankfurt, Germany

https://www.csi-congress.org/frankfurt 
June 29-30, 2021

AI Med Clinical Series: Imaging Focus

Virtual Event

https://ai-med.io/all-events/clinician-series/clinician-serie

s-imaging-focus/

July 8-10, 2021

9th Annual ACHA Conference

Bloomington, $\mathrm{MN}$

https://www.achaheart.org/get-involved/events/2021/9thnational-conference/

July $15-18,2021$

8th Congress of the Asia-Pacific Pediatric Cardiac Society Taipei, Taiwan

http://www.appcs2020.org/

July 23-24, 2021

CICT Chicago 2021

Chicago, USA

https://cictsymposium.com/

September 1-4, 2021

Pediatric Interventional Cardiology Symposium-AICS

Las Vegas, Nevada, USA

http://www.picsymposium.com/

Coming September, 2021

3rd Advanced Fetal Cardiac Course: State of Art and New

Frontiers
October 16, 2021

11th Annual UCLA Fetal Echocardiography Symposium

Virtual event

October 18-12, 2021

American Academy of pediatrics National Conference

Anaheim, California, USA

http://www.aapexperience.org/

October 24-25, 2020

CONGENITAL HEART SURGEONS' SOCIETY Meeting

Chicago, IL

https://meeting.chss.org/

November 13-15, 2021

Scientific Session, American Heart Association

Boston, Massachusetts, USA

http://professional.heart.org

November 1-2, 2021

British Congenital Cardiac Association Annual Conference Birmingham, UK

http://bcca2021.co.uk/

Publisher's Note Springer Nature remains neutral with regard tojurisdictional claims in published maps and institutional afliations. 